\title{
Respiratory comorbidity in South African children with atopic dermatitis
}

\author{
C L Gray, ${ }^{1} \mathrm{MB}$ ChB, FRCPCH, MSc, PhD; M E Levin, ${ }^{1} \mathrm{MB}$ ChB, FCPaeds, PhD; G du Toit, ${ }^{2} \mathrm{MB} \mathrm{ChB}, \mathrm{FRCPCH}$ \\ ${ }^{1}$ Division of Allergology, Department of Paediatrics and Child Health, Red Cross War Memorial Children's Hospital, Cape Town, and \\ Faculty of Health Sciences, University of Cape Town, South Africa \\ ${ }^{2}$ King's Health Partners MRM and Asthma UK Centre in Allergic Mechanisms of Asthma, King's College London, UK; and \\ Department of Paediatric Allergy, Guy's and St Thomas' NHS Foundation Trust, London, UK
}

Corresponding author: C Gray (claudiagray.paediatrics@gmail.com)

Background. Atopic dermatitis (AD) is an early and important step in the propagation of the allergic march, enhancing food and respiratory allergies via epicutaneous sensitisation to allergens.

Objectives. To determine the prevalence and patterns of aeroallergen sensitisation, asthma and allergic rhinitis in South African (SA) children with $\mathrm{AD}$.

Methods. This was a prospective, observational study in a paediatric university hospital in Cape Town, SA. Children with moderate to severe $\mathrm{AD}$, aged 6 months - 10 years, were recruited randomly and investigated for food sensitisation and allergy. They were assessed for sensitisation to aeroallergens by the immuno solid-phase allergen chip test. House-dust mite DerP and DerF, dust mite Eur m, Timothy grass, Bermuda grass, tree pollen, mould (Alternaria), cat and dog sensitisation patterns were analysed. Symptoms of asthma and allergic rhinitis were elicited using a questionnaire modified from the International Study of Asthma and Allergies in Childhood study questions. Results. One hundred participants (59 black Africans and 41 of mixed ethnicity) were enrolled (median age 42 months). Of the participants, $39 \%$ had symptoms of asthma and $53 \%$ symptoms of allergic rhinitis; $89 \%$ tested positive to at least one aeroallergen, most commonly house-dust mite DerP or DerF (81\%), dust mite Eur m (51\%), Timothy grass (36\%) and cat (35\%). Asthma, allergic rhinitis and aeroallergen sensitisation all increased with increasing age, while food allergy decreased with age. Food allergy was not an independent risk factor for respiratory allergies. Children were sensitised to indoor allergens (house-dust mite, pets) from an early age, while pollen allergies increased with age.

Conclusions. In this cohort of SA children with moderate to severe AD, comorbidity with respiratory allergies was high. The prevalence of respiratory allergies increased with age while food allergy decreased with age, in keeping with the pattern of the allergic march. Seasonal allergies increased with age, while house-dust mite and pet allergy peaked in younger children, in keeping with early exposure via a defective skin barrier. Early and effective restoration of the skin barrier in AD may be a target for reducing aeroallergen-related diseases.

S Afr Med J 2017;107(10):904-909. DOI:10.7196/SAMJ.2017.v107i10.12418

Atopic dermatitis (AD) in children has multiple comorbidities and is a major risk factor for propagation of the allergic march, the typical sequence of $\operatorname{IgE}$ responses and clinical symptoms that appear in atopic people. ${ }^{[1]}$ On the basis of longitudinal studies, approximately a third to half of $\mathrm{AD}$ patients will develop asthma, and two-thirds or more will develop allergic rhinitis. ${ }^{[2-7]}$ Epicutaneous sensitisation to aeroallergens has been thought to be responsible, with subsequent migration of sensitised $\mathrm{T}$ cells into the nose and airways, causing upper and lower airway disease. ${ }^{[8,9]}$

Aeroallergen sensitisation is common in AD patients, and in the EPAAC (Early Prevention of Asthma and Allergies in Children) study, $20-40 \%$ of AD patients were sensitised to house-dust mite. ${ }^{[10]}$ The presence of allergic sensitisation to aeroallergens in $\mathrm{AD}$ at 1 year was positively related to the occurrence of asthma. In contrast to food allergen sensitisation, aeroallergen sensitisation continued well beyond the first year of life in the EPAAC cohort. ${ }^{[10]}$ In a 7-year follow-up study of children with $\mathrm{AD}, 80 \%$ of the children became sensitised to airborne allergens and $75 \%$ of them noticed symptoms when exposed. ${ }^{[11]}$

Studies have shown that the severity of AD, as well as comorbidity of $\mathrm{AD}$ and food allergy, are particular risk factors for asthma and allergic rhinitis. ${ }^{[12,13]}$ Food allergy has been found to be an independent risk factor for asthma and allergic rhinitis. Early sensitisation to food allergens, especially hen's egg, has been shown to be a valuable predictor of subsequent sensitisation to inhalant allergens. ${ }^{[2]}$ Coexistence of food allergy with asthma may be a risk factor for hospitalisation for severe asthma exacerbations. ${ }^{[14]}$

In a study looking at the natural history of children with both $\mathrm{AD}$ and food allergy attending a tertiary allergy clinic, $75 \%$ of children had another atopic condition, ${ }^{[13]}$ with $44 \%$ having both allergic rhinitis and asthma, 27\% having allergic rhinitis in isolation and $4 \%$ having asthma in isolation. In children with $\mathrm{AD}$ and egg allergy, respiratory allergies develop in up to $90 \%$ of cases. ${ }^{[15]}$

Respiratory allergies can increase the morbidity in food allergy. Asthma is a risk factor for severe reactions to foods, and a high prevalence of asthma is reported among patients with lifethreatening or fatal food-allergy reactions. ${ }^{[16]}$ Respiratory symptoms as part of a food-allergic reaction are a risk factor for persistence of food allergy. ${ }^{[13]}$

\section{Objectives}

This article explores the comorbidity between AD, food allergy, aeroallergen sensitisation, asthma and allergic rhinitis in South African (SA) children. Age-dependent patterns of aeroallergen sensitisation are explored, and indoor v. outdoor aeroallergens are differentiated with regard to risk profiles for comorbid conditions. 


\section{Methods}

This was part of the wider study investigating food sensitisation and allergy patterns in SA children with $\mathrm{AD}$, including 59 children of black African (Xhosa) origin, and 41 of mixed ethnicity (mixed white and black African). The full methodology has been published previously. ${ }^{[17]}$ The study was approved by the University of Cape Town's Human Research Ethics Committee (ref. no. 426/2009), and fully informed written consent was obtained from the parents/legal guardians.

Only children with $\mathrm{AD}$ were included in this study, and the severity of the $\mathrm{AD}$ was assessed using the SCORAD (SCORing Atopic Dermatitis) index. Participants were assessed for food allergy by questionnaire, skin-prick tests (SPTs), an ImmunoCAP immuno solid-phase allergen chip (ISAC) 103 test and controlled open oral food challenge where indicated.

IgE-mediated food allergy was defined as either:

- a convincing clinical history of significant type I allergic reactions after isolated ingestion of a food during the preceding 6 months, with significantly positive SPT/specific IgE, or

- positive food challenge.

Aeroallergen sensitisation was tested using the ISAC test. Participants were assessed for the following:

- house-dust mite Dermatophagoides pteronyssinus (DerP): components nDer $\mathrm{p} 1$ and nDer $\mathrm{p} 2$

- house-dust mite D. farinae (DerF): nDer f 1 and rDer f 2

- dust mite Euroglyphus maynei (Eur m): rEur m 2

- Bermuda grass: nCyn d 1

- Timothy grass: rPhl p 1, 2, 4, 5, 6, 7, 11 and 12

- tree pollen: olive tree nOle e 1 , nOle e 2 ; plane tree rPla a1, rPla a2; cypress nCup a 1; Japanese cedar nCry j 1; birch rBet v1, 2 and 4; alder rAln $\mathrm{g} 1$

- Alernaria mould: rAlt a 1 and rAlt a 6

- dog: rCan $\mathrm{f} 1$ and 2

- cat: rFel d 1 and 4 .

Symptoms of asthma and allergic rhinitis were elicited using a questionnaire modified from the ISAAC (International Study of Asthma and Allergies in Childhood) study questions: ${ }^{[18]}$

- For asthma: Has your child ever had symptoms of asthma (such as wheeze, persistent cough at night or when exercising, shortness of breath)? If yes, was the asthma diagnosed by a doctor, by a nurse or self-diagnosed?

- For allergic rhinitis: Has your child ever had symptoms of hay fever (such as itchy runny eyes, itchy runny nose, blocked nose, frequent sneezing)? If yes, was the hay fever diagnosed by a doctor, by a nurse or self-diagnosed?

Furthermore, all participants were examined for signs of asthma and allergic rhinitis, and a list of all their preventer and reliever medications for asthma and allergic rhinitis was recorded.

\section{Statistical analysis}

The prevalence of asthma and allergic rhinitis symptoms was determined, and differences between children with and without food allergy were analysed by the $\chi^{2}$ test. Aeroallergen sensitisation patterns were described and analysed for their association with asthma, allergic rhinitis and food allergy.

Since egg allergy has been deemed a major risk factor for respiratory allergies, a separate analysis was performed for egg allergy and asthma, as well as aeroallergen sensitisation patterns.

\section{Results}

\section{Asthma prevalence patterns}

Overall, $39.0 \%$ of the 100 participants described symptoms of asthma, and $29.0 \%$ had doctor-diagnosed asthma. Of those with doctor-diagnosed asthma, 48.3\% (14/29) were on a regular controller and $86.2 \%(25 / 29)$ were on a regular reliever. Asthma prevalence increased significantly with age at the time of study entry: $21.9 \%$ $(7 / 32)$ of children aged $<2$ years, $42.9 \%(12 / 28)$ aged $2-4$ years and $50.0 \%(20 / 40)$ aged $>4$ years had asthma symptoms $(p=0.001)$.

Analysis by eczema severity showed that $30.0 \%(15 / 50)$ of those with moderate eczema (SCORAD 15 - 40) and 28.0\% (14/50) of those with severe eczema (SCORAD $>40$ ) had doctor-diagnosed asthma $(p=0.8)$.

When food allergy was analysed as an independent risk factor for asthma, $35.0 \%(14 / 40)$ of patients with food allergy had doctordiagnosed asthma v. $25.0 \%(15 / 60)$ without food allergy $(p=0.28)$. The most common food allergens associated with asthma were egg and peanut. Of participants with doctor-diagnosed asthma, 37.9\% $(11 / 29)$ had egg allergy and $37.9 \%(11 / 29)$ had peanut allergy.

\section{Allergic rhinitis prevalence patterns}

Of the 100 participants, $53.0 \%$ described symptoms of allergic rhinitis and $28.0 \%$ had doctor-diagnosed allergic rhinitis. Of the 28 with doctor-diagnosed allergic rhinitis, $64.3 \%(18 / 28)$ were on an intranasal corticosteroid and $85.7 \%$ (24/28) were on antihistamines.

Age at study entry was associated with the prevalence of allergic rhinitis: $31.3 \%(10 / 32)$ children aged $<2$ years, $53.6 \%(15 / 28)$ aged 2 - 4 years and $70.0 \%(28 / 40)$ aged $>4$ years had allergic rhinitis symptoms.

Analysis by eczema severity showed that $28.0 \%(14 / 50)$ of those with moderate eczema (SCORAD 15 - 40) and 28.0\% (14/50) of those with severe eczema (SCORAD $>40$ ) had doctor-diagnosed allergic rhinitis $(p=1)$.

In the analysis of food allergy as an independent risk factor for allergic rhinitis, $27.5 \%(11 / 40)$ of children with food allergy and $28.3 \%(17 / 60)$ without food allergy had doctor-diagnosed allergic rhinitis $(p=0.93)$.

Age-related changes in food allergy, asthma and allergic rhinitis prevalence are shown in Fig. 1.

\section{Sensitisation pattern to aeroallergens}

Overall, $89.0 \%$ of the 100 children tested positive to at least one aeroallergen on the ISAC test. The median number of positive aeroallergen tests was 3.5. Aeroallergen sensitisation was most common to house-dust mite DerP at $81.0 \%$, followed by DerF at $78.0 \%$. Aeroallergen sensitisation patterns are depicted in Table 1 and Fig. 2. Sensitisation to tree pollen, cat dander, Bermuda grass and dog

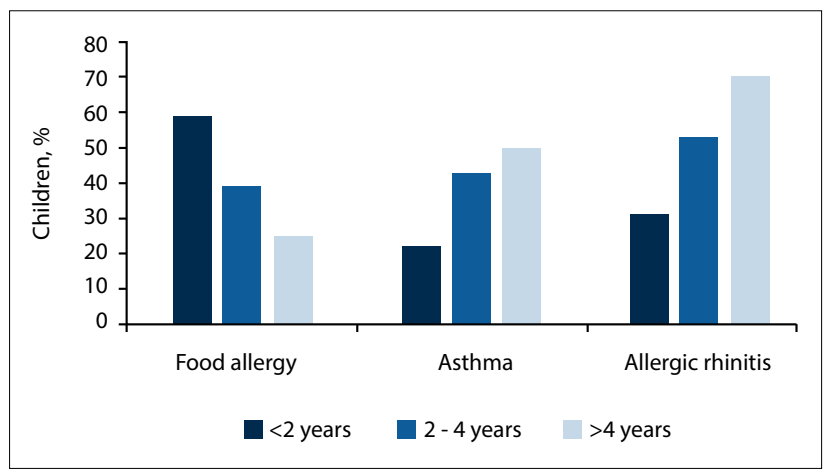

Fig. 1. Food allergy, asthma and allergic rhinitis prevalence by age group. 


\begin{tabular}{|c|c|c|c|c|}
\hline & $\begin{array}{l}\text { Sensitised overall } \\
(N=100)\end{array}$ & $\begin{array}{l}\text { Xhosas sensitised } \\
(N=59)\end{array}$ & $\begin{array}{l}\text { Mixed ethnicity } \\
\text { sensitised }(N=41)\end{array}$ & $\begin{array}{l}\text { Interethnic difference } \\
(p \text {-value })^{*}\end{array}$ \\
\hline Any aeroallergen sensitisation & 89.0 & 86.4 & 92.7 & 0.33 \\
\hline House-dust mite DerP & 81.0 & 79.7 & 82.9 & 0.68 \\
\hline House-dust mite DerF & 78.0 & 77.9 & 78.0 & 0.99 \\
\hline Dust mite Eur m & 51.0 & 54.2 & 46.3 & 0.51 \\
\hline Timothy grass & 36.0 & 28.8 & 46.3 & 0.08 \\
\hline Cat & 35.0 & 27.1 & 46.3 & $0.05^{+}$ \\
\hline Bermuda grass & 30.0 & 20.3 & 3.9 & $0.011^{\dagger}$ \\
\hline Dog & 18.0 & 10.2 & 29.2 & $0.014^{\dagger}$ \\
\hline Tree pollen & 17.0 & 10.2 & 26.8 & $0.03^{+}$ \\
\hline Alternaria mould & 16.0 & 13.6 & 19.5 & 0.42 \\
\hline
\end{tabular}

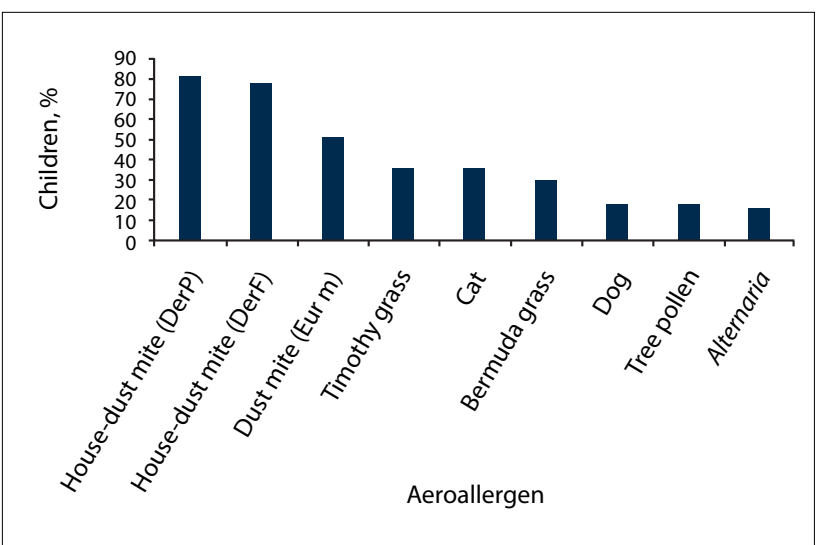

Fig. 2. Prevalence of aeroallergen sensitisation by the immuno solid-phase allergen chip test in children with atopic dermatitis $(\mathrm{N}=100)$.

dander was significantly more common in the mixed-race group than in the Xhosa group.

\section{Sensitisation patterns to individual allergens \\ House-dust mite sensitisation}

DerP. Overall, $81.0 \%$ of children were sensitised to DerP. There was no significant increase in asthma or allergic rhinitis symptoms in children with DerP sensitisation (Table 2). There was, however, a significant association between DerP sensitisation and food allergy: 92.5\% (37/40) of children with food allergy were DerP sensitised v. $73.3 \%$ (44/60) without food allergy $(p=0.02)$.

DerF. Overall, $78.0 \%$ of children were sensitised to DerF. There was no significant increase in asthma or allergic rhinitis symptoms in children with DerF sensitisation (Table 2). There was, however, a significant association between DerF sensitisation and food allergy: $90.0 \%(36 / 40)$ of children with food allergy were DerF sensitised v. $70.0 \%(42 / 60)$ without food allergy $(p=0.02)$.

Of the children sensitised to house-dust mite, $95.0 \%$ were sensitised to both DerP and DerF, so there was a near-complete overlap between house-dust mite species.

Eur m. Overall $51.0 \%$ of children were sensitised to the dust mite Eur m. Fifty of the 51 children with Eur m sensitisation (98.0\%) were co-sensitised with DerP. There was a significant increase in Eur m sensitisation among asthmatics: $66.7 \%(26 / 39)$ of children with asthma symptoms were Eur m sensitised v. $41.0 \%$ (25/61) of nonasthmatics $(p=0.015)$ (Table 2). There was no significant association between Eur $\mathrm{m}$ sensitisation and food allergy.

\section{Timothy grass sensitisation}

Overall, $36.0 \%$ of children were sensitised to at least one of the Timothy grass antigens, and $83.3 \%$ (30/36) of children with Timothy grass sensitisation were also Bermuda grass sensitised.

There was a significant increase in Timothy grass sensitisation among asthmatics: $51.3 \%$ (20/39) of children with asthma symptoms were Timothy grass sensitised v. $26.2 \%(16 / 61)$ of non-asthmatics $(p=0.008)$. Timothy grass sensitisation was also significantly increased in allergic rhinitis sufferers: $51.3 \%$ (27/53) of children with symptoms of allergic rhinitis were Timothy grass sensitised v. $19.1 \%$ $(9 / 47)$ without allergic rhinitis symptoms $(p=0.001)$ (Table 2). There was no significant association between Timothy grass sensitisation and food allergy.

\section{Bermuda grass sensitisation}

Overall, $30.0 \%$ of children were Bermuda grass sensitised. All children with Bermuda grass sensitisation were also Timothy grass sensitised.

There was a significant increase in Bermuda grass sensitisation among asthmatics: $46.2 \%$ (18/39) of children with asthma symptoms were Bermuda grass sensitised v. $19.7 \%(12 / 61)$ of non-asthmatics $(p=0.005)$. Bermuda grass sensitisation was also significantly increased in allergic rhinitis sufferers: $43.4 \%$ (23/53) of children with symptoms of allergic rhinitis were Bermuda grass sensitised v. $14.9 \%$ (7/47) without allergic rhinitis symptoms $(p=0.002)$ (Table 2). There was no significant association between Bermuda grass sensitisation and food allergy.

\section{Cat sensitisation}

Overall, $35.0 \%$ of children were sensitised to at least one of the cat allergens tested.

There was no significant increase in asthma or allergic rhinitis symptoms in children with cat sensitisation (Table 2). There was, however, a significant association between cat sensitisation and food allergy: $57.5 \%(23 / 40)$ of children with food allergy were cat sensitised v. $20.0 \%(12 / 60)$ of those without food allergy $(p<0.001)$.

\section{Dog sensitisation}

Overall, $18.0 \%$ of children were dog sensitised, and 77.8\% (14/18) of children with dog sensitisation were also cat sensitised.

Dog sensitisation was not significantly associated with asthma or allergic rhinitis symptoms (Table 2). However, there was a significant association between dog sensitisation and food allergy: $37.5 \%(15 / 40)$ of children with food allergy were dog sensitised v. 5.0\% (3/60) of those without food allergy $(p<0.001)$. 
Table 2. Aeroallergen sensitisation patterns in children with concomitant asthma and allergic rhinitis, \%

\begin{tabular}{|c|c|c|c|c|c|c|}
\hline Sensitisation & $\begin{array}{l}\text { Patients with } \\
\text { asthma }(N=39)\end{array}$ & $\begin{array}{l}\text { Patients without } \\
\text { asthma }(N=61)\end{array}$ & $\begin{array}{l}\text { Difference } \\
(p \text {-value })^{*}\end{array}$ & $\begin{array}{l}\text { Patients with } \\
\text { allergic rhinitis } \\
(N=53)\end{array}$ & $\begin{array}{l}\text { Patients without } \\
\text { allergic rhinitis } \\
(N=47)\end{array}$ & $\begin{array}{l}\text { Difference } \\
(p \text {-value })^{*}\end{array}$ \\
\hline Any aeroallergen & 92.3 & 86.9 & 0.4 & 90.6 & 87.2 & 0.6 \\
\hline House-dust mite DerP & 87.2 & 77.0 & 0.21 & 83.0 & 78.7 & 0.59 \\
\hline House-dust mite DerF & 82.1 & 75.4 & 0.43 & 83.0 & 72.3 & 0.2 \\
\hline Dust mite Eur m & 66.7 & 42.6 & $0.015^{\dagger}$ & 60 & 40.4 & 0.06 \\
\hline Timothy grass & 51.3 & 26.2 & $0.008^{\dagger}$ & 50.9 & 19.1 & $0.001^{\dagger}$ \\
\hline Cat & 41.0 & 31.1 & 0.31 & 35.8 & 34.0 & 0.85 \\
\hline Bermuda grass & 46.2 & 19.7 & $0.005^{\dagger}$ & 43.4 & 14.9 & $0.002^{\dagger}$ \\
\hline Dog & 23.1 & 14.8 & 0.3 & 18.9 & 17.0 & 0.81 \\
\hline Tree pollen & 25.6 & 11.5 & 0.07 & 22.6 & 10.6 & 0.111 \\
\hline Alternaria & 23.1 & 11.5 & 0.12 & 13.2 & 19.1 & 0.42 \\
\hline
\end{tabular}

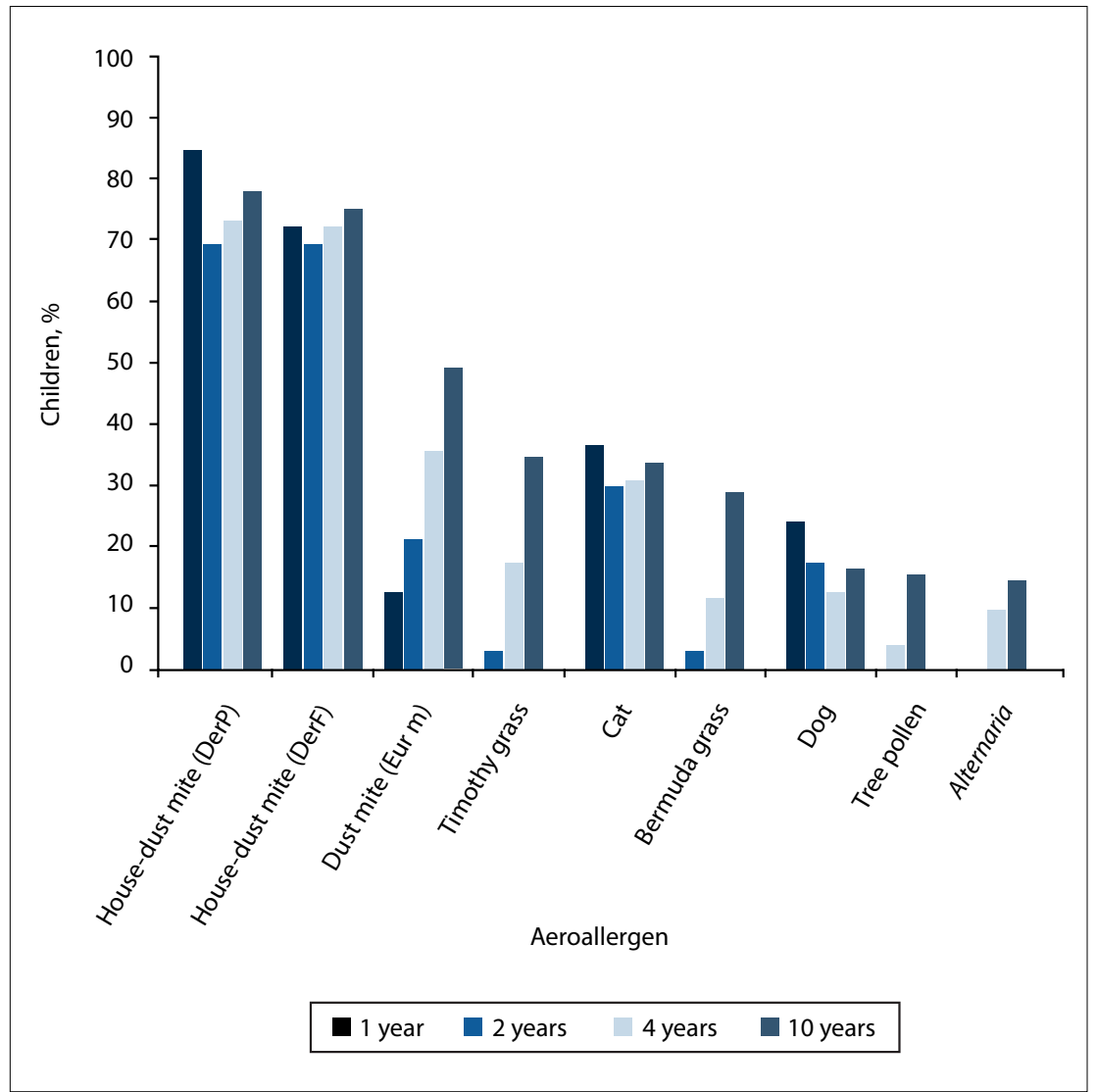

Fig. 3. Aeroallergen sensitisation at different ages.

\section{Tree pollen sensitisation}

Overall, $17.0 \%$ of children were sensitised to any of the tree pollens tested. Tree pollen sensitisation was not significantly associated with asthma, allergic rhinitis or food allergy in this cohort.

\section{Alternaria mould sensitisation}

Overall, $16.0 \%$ of children were Alternaria mould sensitised. Alternaria sensitisation was not significantly associated with asthma, allergic rhinitis or food allergy. at 1 year of age) compared with the older children, suggesting that sensitisation to these allergens occurs early in life. Timothy grass, Bermuda grass and tree pollen sensitisation increased with age, suggesting an ongoing sensitisation process to certain aeroallergens far beyond the first year of life. The mean numbers of aeroallergens children were sensitised to were 2.25 in those aged $<2$ years, 2.8 in those aged $\leq 4$ years and 3.63 in those aged $\leq 10$ years. This suggests that aeroallergen sensitisation may continue beyond 4 years of age.

\section{Egg allergy and respiratory comorbidity}

There was no significant difference in asthma and allergic rhinitis prevalence between children with or without egg allergy. Of children with egg allergy, $44.0 \%$ (11/25) had symptoms of asthma, compared with $37.3 \%(28 / 75)$ without egg allergy $(p=0.55)$. There was a tendency for older children ( $\geq 24$ months) with egg allergy to have more asthma than younger children with egg allergy, but this did not reach statistical significance: $31.3 \%(5 / 16)$ of children aged $<24$ months with egg allergy had asthma symptoms, v. $66.7 \%(6 / 9)$ of children aged $\geq 24$ months $(p=0.08$ ).

\section{Discussion}

The significant comorbidity between AD, food allergy, asthma and allergic rhinitis suggests that the broken epithelial barrier in patients with $\mathrm{AD}$ is a major risk factor for epicutaneous sensitisation to allergens. Allergic comorbidity was significant in our cohort of children with moderate to severe $\mathrm{AD}$. The prevalence of asthma symptoms, at $39 \%$ in this selected population, is significantly higher than in the general population, estimated at $14 \%$ in SA adolescents 
Table 3. Aeroallergen sensitisation by age, $\%$

\begin{tabular}{|c|c|c|c|c|}
\hline & \multicolumn{4}{|c|}{ Age (years) } \\
\hline & $\begin{array}{l}\leq 1 \\
(N=8)\end{array}$ & $\begin{array}{l}\leq 2 \\
(N=32)\end{array}$ & $\begin{array}{l}\leq 4 \\
(N=60)\end{array}$ & $\begin{array}{l}\leq 10 \\
(N=100)\end{array}$ \\
\hline Any aeroallergen & 87.5 & 78.1 & 85.0 & 89.0 \\
\hline House-dust mite DerP & 87.5 & 71.9 & 76.7 & 81.0 \\
\hline House-dust mite DerF & 75.0 & 71.9 & 75.0 & 78.0 \\
\hline Dust mite Eur m & 12.5 & 21.9 & 36.7 & 51.0 \\
\hline Timothy grass & 0 & 3.1 & 18.3 & 36.0 \\
\hline Cat & 37.5 & 31.3 & 31.7 & 35.0 \\
\hline Bermuda grass & 0 & 3.1 & 11.7 & 30.0 \\
\hline Dog & 25.0 & 18.8 & 15.0 & 18.0 \\
\hline Tree pollen & 0 & 0 & 5.0 & 17.0 \\
\hline Alternaria & 0 & 0 & 10.0 & 16.0 \\
\hline
\end{tabular}

based on data from the ISAAC study. ${ }^{[18]}$ Although the ISAAC study was based on adolescents, these are the only data for respiratory allergies that we have from a large unselected population in SA. The $53 \%$ prevalence of reported allergic rhinitis symptoms in our selected population is higher than the figure of $39 \%$ in SA adolescents in the ISAAC study. There were no significant ethnic differences in asthma and allergic rhinitis prevalence, and no significant difference between food-allergic and food-tolerant children in terms of asthma and allergic rhinitis prevalence. Overall, egg sensitisation and allergy rates were not associated with an increase in asthma or allergic rhinitis symptoms. AD per se rather than food allergy therefore seems to be the more important risk factor for progression to respiratory diseases in this cohort.

The prevalence of both asthma and allergic rhinitis increased with age, in contrast to the prevalence of food allergy, which fell with age. This reflects the progression of the allergic march, with eczema and food allergy peaking earlier than asthma and allergic rhinitis.

Aeroallergen sensitisation was extremely common in this population; overall $89 \%$ of children with $\mathrm{AD}$ were sensitised to at least one aeroallergen. The pattern was age dependent. Sensitisation to indoor allergens such as house-dust mite DerP and DerF and cat and dog dander had peaked by the age of 2 years, whereas sensitisation to grass pollen, tree pollen and Alternaria mould increased with age. Unlike food allergy sensitisation, which peaked by 2 years and then declined steadily, aeroallergen sensitisation occurred well after 2 years. If negative at a young age, an aeroallergen test should therefore be repeated at an older age in high-risk patients. Also, the aeroallergen pattern evolves over time, with the seasonal allergens becoming more important in older children. This can act as a guide to the prudent choice of aeroallergen tests according to age group.

Sensitisation to Timothy grass, Bermuda grass and dust mite Eur $\mathrm{m}$ was significantly higher in asthmatic than non-asthmatic children, and sensitisation to Timothy and Bermuda grass was significantly higher in children with allergic rhinitis symptoms. The indoor allergens DerP, DerF, cat and dog were not significantly higher in asthma or AR. It may be that the induction of specific IgE responses to certain aeroallergens and the development of childhood asthma are at least partly determined by independent factors. ${ }^{[19]}$

Sensitisation to the indoor allergens DerP, DerF, cat and dog was, however, significantly higher in children with food allergy. As these are the allergens that peak in sensitisation at a young age, this may reflect early-onset epicutaneous sensitisation to a number of food and aeroallergens with which the young child is in contact in his or her initial predominantly indoor environment.
Certain allergens showed significant overlap in this cohort: $99 \%$ of children with DerF sensitisation were also sensitised to DerP, and $100 \%$ of children with Bermuda grass sensitisation were also sensitised to Timothy grass. This could aid in reducing an aeroallergen panel in children even further by choosing DerP and Timothy grass as the representative allergens for house-dust mite and grass allergy, respectively. This could be particularly useful in a resource-poor setting, or in a setting in which a reduced SPT panel size is desirable (e.g. in a very anxious or young child).

Poor control of one atopic condition can lead to increased morbidity in another atopic condition, e.g. poor asthma control is a significant risk factor for more severe food-allergic reactions. ${ }^{[16]}$ This is particularly concerning in light of the low rates of controller medication use reported by asthmatic children in this cohort: only $48 \%$ of patients with doctor-diagnosed asthma took a regular controller.

Improved control of atopic conditions may reduce the progression to or severity of associated atopic conditions, e.g. better control of $\mathrm{AD}$ may reduce the risk of food allergy or asthma. An Italian study ${ }^{[20]}$ suggested that early diagnosis and improved management of AD may lead to a reduction in the percentage of children evolving towards asthma from $29 \%$ to $15 \%$. Integrated management of the allergic patient is therefore essential, treating each allergic manifestation well to try to reduce progression to or severity of associated allergic manifestations.

\section{Study limitations}

Limitations of this study include a relatively small sample size of 100. Moreover, the fact that this was a highly selected population of patients with $\mathrm{AD}$ means that results are not generalisable to an unselected population.

\section{Conclusions}

In this cohort of SA children with moderate to severe $\mathrm{AD}$, comorbidity with respiratory allergies was high: $89 \%$ of children were sensitised to at least one aeroallergen (most commonly housedust mite), 39\% had symptoms of asthma and 53\% had symptoms of allergic rhinitis. The prevalence of asthma and allergic rhinitis increased with age, while food allergy decreased with age, in keeping with the pattern of the allergic march. Food allergy was not an independent risk factor for respiratory allergies in this cohort. The aeroallergen pattern evolved over time, with the seasonal allergens becoming more important in the older children and house-dust mite and pet allergy peaking in younger children, in parallel with the peak in food allergy. These findings can help guide the prudent choice of aeroallergen tests according to age group. As aeroallergen sensitisation may occur via the epicutaneous route in children with $\mathrm{AD}$, early and effective restoration of the skin barrier may be a target for reducing aeroallergen-related diseases.

Acknowledgements. We thank Thermo Fisher for providing reagents for the ISAC testing for this study.

Author contributions. CG was responsible for the original research, supervised by ML and GdT; CG wrote the article; and ML and GdT checked the article.

Funding. The study was part-funded by a research grant from the Allergy Society of South Africa.

Conflicts of interest. None.

1. Silverberg II, Simpson EL. Association between severe eczema in children and multiple co-morbic
conditions and increased healthcare utilisation. Pediatr Allergy Immunol 2013;24(5):476-481. https:// conditions and increased he
doi.org/10.1111/pai.12095 
2. Van der Hulst AE, Klip H, Brand PL. Risk of developing asthma in young children with atopic eczema: A systematic review. J Allergy Clin Immunol 2007;120(3):565-569. https://doi.org/10.1016/j. jaci.2007.05.042

3. Zheng T, Yu J, Oh MH, Zhu Z. The atopic march: Progression from atopic dermatitis to allergic rhinitis and asthma. Allergy Asthma Immunol Res 2011;3(2):67-73. https://doi.org/10.4168/aair.2011.3.2.67 4. Pasternack B. The prediction of asthma in infantile eczema. J Pediatr 1965;66(Suppl):164-165. https:// doi.org/10.1016/s0022-3476(65)80273-6

5. Stifler WC. A twenty-one year follow-up of infantile eczema. J Pediatr 1965;66(Suppl):166-167. https:// doi.org/10.1016/s0022-3476(65)80274-8

6. Wuthrich B, Schmid-Grendelmeier P. Natural course of AEDS. Allergy 2002;57(3):267-268. https:// doi.org/10.1034/j.1398-9995.2002.1n3572.x

7. Spergel JM. From atopic dermatitis to asthma: The atopic march. Ann Allergy Asthma Immunol 2010;105(2):99-106. https://doi.org/10.1016/j.anai.2009.10.002

8. He R, Kim HY, Yoon J, et al. Exaggerated IL-17 response to epicutaneous sensitisation mediates airway inflammation in the absence of IL-4 and IL-13. J Allergy Clin Immunol 2009;124(4):761-770. https:// doi.org/10.1016/j.jaci.2009.07.040

9. Gustafsson D, Sjöberg O, Foucard T. Sensitization to food and airborne allergens in children with atopic dermatitis followed up to 7 years of age. Pediatr Allergy Immunol 2003;14(6):448-452. https:// doi.org/10.1046/j.0905-6157.2003.00093.x

10. De Benedictis FM, Franceschini F, Hill D, et al. The allergic sensitization in infants with atopic eczema from different countries. Allergy 2009;64(2):295-303. https://doi.org/10.1111/j.13989995.2008.01779.x

11. Kulig M, Bergmann R, Niggemann B, et al., for the MAS Study Group. Prediction of sensitization to inhalant allergens in childhood: Evaluating family history, atopic dermatitis and sensitizatio to food allersen
12. Illi S, von Mutius E, Lau S, et al. The natural course of atopic dermatitis from birth to age 7 years and the association with asthma. J Allergy Clin Immunol 2004;113(5):925-931. https://doi.org/10.1016/j. jaci.2004.01.778

13. Sampson HA, Scanlon SM. Natural history of food hypersensitivity in children with atopic dermatitis. Pediatr 1989;115(1):23-27. https://doi.org/10.1016/s0022-3476(89)80323-3

14. Vogel NM, Katz HT, Lopez R, et al. Food allergy is associated with potentially fatal childhood asthma. J Asthma 2008;45(10):862-866. https://doi.org/10.1080/02770900802444195

15. Spergel JM, Paller AS. Atopic dermatitis and the atopic march. J Allergy Clin Immunol 2003;112(6 Suppl):S118-S127. https://doi.org/10.1016/j.jaci.2003.09.033

16. Bock SA, Munoz-Furlong A, Sampson HA. Fatalities due to anaphylactic reactions. J Allergy Clin Immunol 2001;107(1):191-193. https://doi.org/10.1067/mai.2001.112031

17. Gray CL, Levin ME, du Toit G. Food allergy in South African children with atopic dermatitis. Pediatr Allergy Immunol 2014;25(6):572-579. https://doi.org/10.1111/pai.12270

18. Zar HJ, Ehrlich RI, Workman L, Weinberg EG. The changing prevalence of asthma, allergic rhinitis and atopic eczema in African adolescents from 1995 to 2002. Pediatr Allergy Immunol 2007;18(7):560-565. https://doi.org/10.1111/j.1399-3038.2007.00554.x

19. Lau S, Sommerfeld C, Niggemann B, et al. Early exposure to house-dust mite and cat allergens and development of childhood asthma: A cohort study. Multicentre Allergy Study Group. Lancet 2000;356(9239):1392-1397. https://doi.org/10.1016/s0140-6736(00)02842-7

20. Ricci G, Patrizi A, Giannetti A, et al. Does improvement in management of atopic dermatitis influence the appearance of respiratory allergic diseases? A follow-up study. Clin Mol Allergy 2010;8:8. https:// doi.org/10.1186/1476-7961-8-8

Accepted 15 May 2017. 